\title{
BROOKHTUEN
}

NATIONAL LABORATORY

BNL-90848-2009-CP

\section{Directional Stand-off Detection of Fast Neutrons and Gammas Using Angular Scattering Distributions}

\author{
Peter E. Vanier, Istvan Dioszegi, Cynthia Salwen and Leon \\ Forman
}

Presented at the IEEE Nuclear Science Symposium

Orlando, Florida

October 25-31, 2009

December 2009

\author{
Nonproliferation and National Security Department \\ Detector Development and Testing Division \\ Brookhaven National Laboratory \\ P.O. Box 5000 \\ Upton, NY 11973-5000 \\ www.bnl.gov
}

Notice: This manuscript has been authored by employees of Brookhaven Science Associates, LLC under Contract No. DE-AC02-98CH10886 with the U.S. Department of Energy. The publisher by accepting the manuscript for publication acknowledges that the United States Government retains a non-exclusive, paid-up, irrevocable, world-wide license to publish or reproduce the published form of this manuscript, or allow others to do so, for United States Government purposes. 
This preprint is intended for publication in a journal or proceedings. Since changes may be made before

publication, it may not be cited or reproduced without the author's permission.

\section{DISCLAIMER}

This report was prepared as an account of work sponsored by an agency of the United States Government. Neither the United States Government nor any agency thereof, nor any of their employees, nor any of their contractors, subcontractors, or their employees, makes any warranty, express or implied, or assumes any legal liability or responsibility for the accuracy, completeness, or any third party's use or the results of such use of any information, apparatus, product, or process disclosed, or represents that its use would not infringe privately owned rights. Reference herein to any specific commercial product, process, or service by trade name, trademark, manufacturer, or otherwise, does not necessarily constitute or imply its endorsement, recommendation, or favoring by the United States Government or any agency thereof or its contractors or subcontractors. The views and opinions of authors expressed herein do not necessarily state or reflect those of the United States Government or any agency thereof. 


\title{
Directional Stand-off Detection of Fast Neutrons and Gammas Using Angular Scattering Distributions
}

\author{
Peter E. Vanier, Member, IEEE, Istvan Dioszegi, Cynthia Salwen, and Leon Forman
}

\begin{abstract}
We have investigated the response of a Double Scatter Neutron Spectrometer (DSNS) for sources at long distances ( $>200$ meters). We find that an alternative method for analyzing double scatter data avoids some uncertainties introduced by amplitude measurements in plastic scintillators. Time of flight is used to discriminate between gamma and neutron events, and the kinematic distributions of scattering angles are assumed to apply. Non-relativistic neutrons are most likely to scatter at $45^{\circ}$, while gammas with energies greater than $2 \mathrm{MeV}$ are most likely to be forward scattered. The distribution of scattering angles of fission neutrons arriving from a distant point source generates a $45^{\circ}$ cone, which can be back-projected to give the source direction. At the same time, the distribution of Compton-scattered gammas has a maximum in the forward direction, and can be made narrower by selecting events that deposit minimal energy in the first scattering event. We have further determined that the shape of spontaneous fission neutron spectra at ranges $>110 \mathrm{~m}$ is still significantly different from the cosmic ray background.
\end{abstract}

\section{INTRODUCTION}

$\mathrm{D}$ ETECTION of radiation sources at long stand-off distances requires large area, low cost sensors that can maximize the subtended solid angle. Plastic scintillator detectors are sensitive to both gammas and fast neutrons and can be manufactured in large areas for relatively low cost. Individual detectors, in the shape of thick rectangular plates optically coupled to photomultipliers, are not normally sensitive to the direction of incident neutron trajectories. However, when arranged in two planes and coupled to high-bandwidth coincidence-timing electronics they can be used to create a detection system that measures both the direction and the energy spectrum of the fast neutrons, and distinguish them from gamma rays by time of flight [1-4].

In the conventional method of data analysis, the angle of scatter of a neutron is estimated using the kinematic equations with measured values for the energy deposited in the first plane and the time of flight between the planes. The resulting images are limited in precision by the statistical uncertainty of total amplitude of the small number of optical photons collected per event, as well as by the uncertainty in the time of flight and in the location of an event in each plane.

We now discuss a different method of analysis, based entirely on the locations of events, in which it is not necessary

Manuscript received November 9, 2009. This work was supported in part by the U.S. Department of Energy Contract No. DE-AC02-98CH10886 .

Peter E. Vanier, Istvan Dioszegi, and Cynthia Salwen are with Brookhaven National Laboratory, Upton, NY 11973 USA (telephone: 631-344-3535, email: vanier@bnl.gov).

Leon Forman is with Ion Focus Technology, Miller Place, NY 11764 USA. (telephone: 631-344-5852, e-mail: 1forman@bnl.gov). to make an amplitude measurement. Instead, we make use of the scattering angle probability distribution, which peaks at 45 degrees for neutrons scattering in hydrogenous material. An analogous method can be used to extract directional information from Compton scattered gamma rays, which have an angular distribution that becomes forward directed at relativistic electron energies, above $2 \mathrm{MeV}$. Even though plastic scintillators do not have sufficient energy resolution to work effectively as a Compton imager, the method allows us to determine the direction to a point source of gammas. The detector system therefore provides two independent estimates of the directions to a gamma source and a neutron source that may be, but are not necessarily, co-located. We have demonstrated these methods with measurements of a source at distances over 200 meters.

We have investigated the detector response for $252 \mathrm{Cf}$ neutron spectra transmitted through air compared to that for cosmic-ray background, which determines the limit of standoff range. We have compared the detector signal-tonoise ratio with that for a moderated ${ }^{3} \mathrm{He}$ detector, the 8.2" diameter Bonner sphere. This geometry is the optimal diameter for detection of fission energy neutrons. We will show that the double scatter technique can maximize the signal-to-background by limiting the energy analysis window to fission neutrons $(2-7 \mathrm{MeV})$ and by limiting the accepted neutron pulses to the forward (positive TOF) direction.

\section{PRIOR WORK}

The equipment employed in this work has been previously described [2-4], and it bears some similarity to other systems developed for warhead counting [5] or for basic nuclear physics [6]. Other groups have also used liquid scintillators with pulse shape discrimination of neutrons from gammas $[7,8]$. In all these publications, the direction of the incident neutron is obtained by estimating the energy deposited in the first scattering event by the integrated scintillation light and the subsequent energy of the scattered neutron by time of flight between two separated detectors. The kinematic equations are then used to calculate the angle of scattering of the neutron that can be back-projected as a cone whose axis is the scattered neutron vector. A series of overlapping cones generated by multiple events can be added to provide an image with a dominant bright spot at the source direction. One drawback of this method is that the optical response of the organic scintillator is non-linear with the energy of the recoil proton. Another problem is that each scintillation event generates only a small number of photoelectrons in the 
photomultiplier tubes (PMTs), causing statistical fluctuations in the measurement of deposited energy the time of flight.

The general approach for maximizing the detector standoff range was outlined in a previous paper [10]. It is based on the fact that the cosmic-ray neutron spectrum is distributed over a large energy range $\left(10^{-8} \mathrm{eV}-1000 \mathrm{GeV}\right)$ whereas spontaneous fission neutrons are emitted in the range $2-7 \mathrm{MeV}$. In this set of measurements, the range in air was influenced by transmission, with a mean free path of $120 \mathrm{~m}$, as well as random coincidences between gamma ray events.

\section{HARDWARE}

The 8-paddle plastic scintillator detector that was previously described [2-4] was mounted in a small truck as shown in the inset of Fig. 1. The truck was parked at a series of distances from the source in the calibration facility, a square building on the right. The ${ }^{252} \mathrm{Cf}$ source strength was $\sim 2 \times 10^{8}$ neutrons per second when the source was exposed from its shield. The data acquisition system consisted of VME modules that recorded the amplitude and time of each photomultiplier pulse, provided that a pulse occurred in both the front plane of paddles and the back plane within a coincidence window of 100 ns.

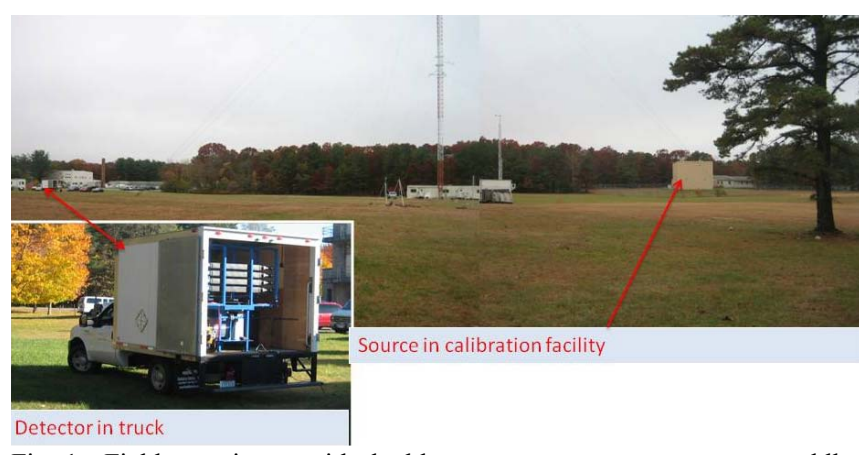

Fig. 1. Field experiment with double-scatter neutron spectrometer paddle detector mounted in a small truck parked at a series of distances from the source in the calibration facility on the right. The source strength was $10^{8}$ neutrons per second.

\section{SOFTWARE}

The data were acquired using LabView ${ }^{\mathrm{TM}}$ software that recorded each set of raw event data to be post-processed by a separate routine. Eventually, the two parts of the software were combined, so that each event could be plotted in an image "on the fly" provided that the appropriate choices for data cuts were made in advance. In this paper we focus on how those images are constructed. We start by plotting the directions of vectors connecting the event in the front plane with the event in the back plane, producing images of the scatter angles.

\section{EXPERIMENTAL RESULTS}

\section{A. Gamma directions}

Although the plastic scintillators do not provide adequate energy resolution to be used as a high precision Compton camera, and do not generally collect the full energy of the gamma rays, they still can be used to estimate a rough pointing direction, which may be all that is needed in some scenarios. In order to do this, events were selected in which only a small fraction of the energy was deposited in the first scattering event but a large amount was detected in the second plane. An additional condition was imposed to select events with a time of flight less than $5 \mathrm{~ns}$ so as to exclude the neutrons. This selection gives images such as that in Fig. 2, which shows a definite bright spot near the center. Thus, for the selected gamma events, the scatter angles point towards the source. The main limitation of our system in using this method is its insensitivity to the lowest energy events.

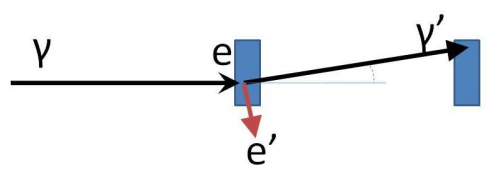

Fig. 2. Using the double scatter geometry, gammas are selected by time of flight in the range $0-5 \mathrm{~ns}$ for interplanar spacing of $50 \mathrm{~cm}$. Events with low scattering angles are emphasized by imposing an upper limit on the amplitude in the first plane and a lower limit on the amplitude in the second plane. The image shows the distribution of scattering angles over a field of view of $\pm 60^{\circ}$. The vertical modulation corresponds to the discrete positions of the paddles.

A horizontal profile of the gamma image intensity obtained in this way can be compared with the profiles calculated by the Compton scattering formula for suitable choices of photon energy, as shown in Fig. 3. It is clear that without resorting to the construction of a high-precision multilayer Compton camera design, one can easily gain directional information with a low-cost large-area plastic scintillator detector if configured for double scatter. The data in Figs. 2 and 3 were acquired at a range of $33 \mathrm{~m}$ from the source.

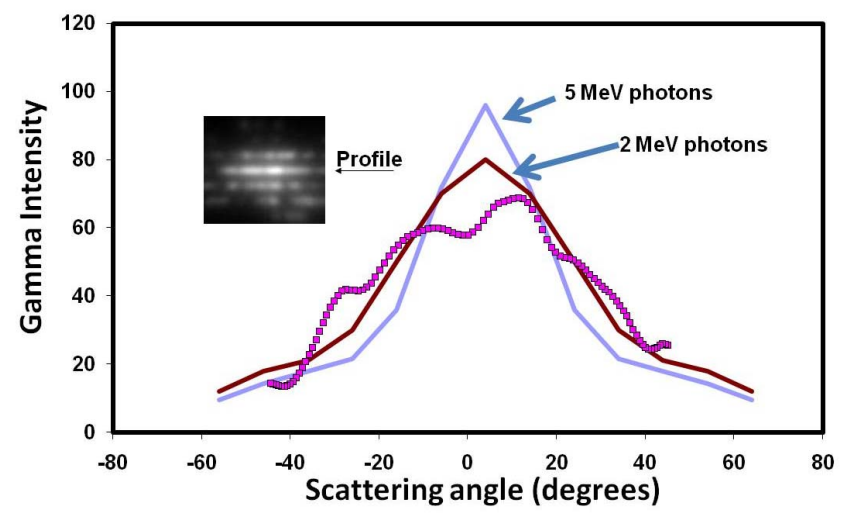

Fig. 3. Horizontal profile of Compton scattering angle distribution compared to theory. 


\section{B. Neutron directions}

In order to select neutron events, we cut the events to include only those with time of flight in the range 10-35 ns. For the interplanar spacing of $50 \mathrm{~cm}$, this range covers the energies of the fission spectrum neutrons. The image in Fig. 4 shows experimental data acquired with the source at a distance of $33 \mathrm{~m}$, in which each scattered neutron vector is projected onto a plane as a spot with a Lorentzian intensity profile having a width corresponding to the estimated error in the position. These fuzzy spots combine to form an ellipse that characterizes the most likely scattering angles. There is also some vertical modulation corresponding to the discrete positions of the paddles.
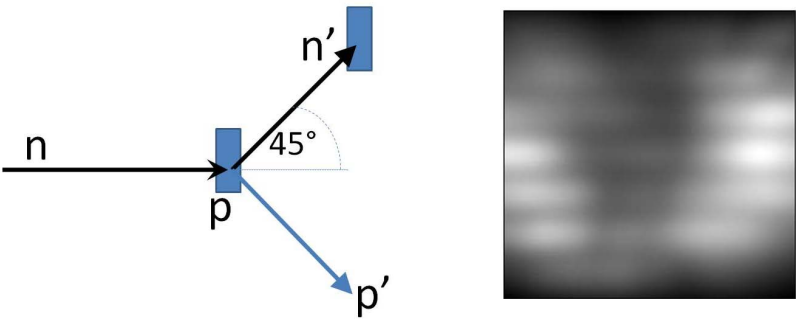

Fig. 4. Scattering angles from a point source of non-relativistic neutrons are predominantly at $45^{\circ}$ from the incident vector. Neutrons are selected by time of flight in the range 10-35 ns for interplanar spacing of $50 \mathrm{~cm}$.

The horizontal profile through the center of the image is shown in Fig. 5, and compared with the distribution expected from kinematic theory. The main difference is that at the center of the image (scattering angle $=0$ ) one would expect the neutron intensity to be zero. Very low-angle neutron scattering events would not be recorded as coincidences, because the first event would deposit energies below the detectable threshold. We attribute the non-zero intensity at the center of the ellipse to accidental coincidences from uncorrelated background gamma rays that fall within the time-of-flight window for fission neutrons.

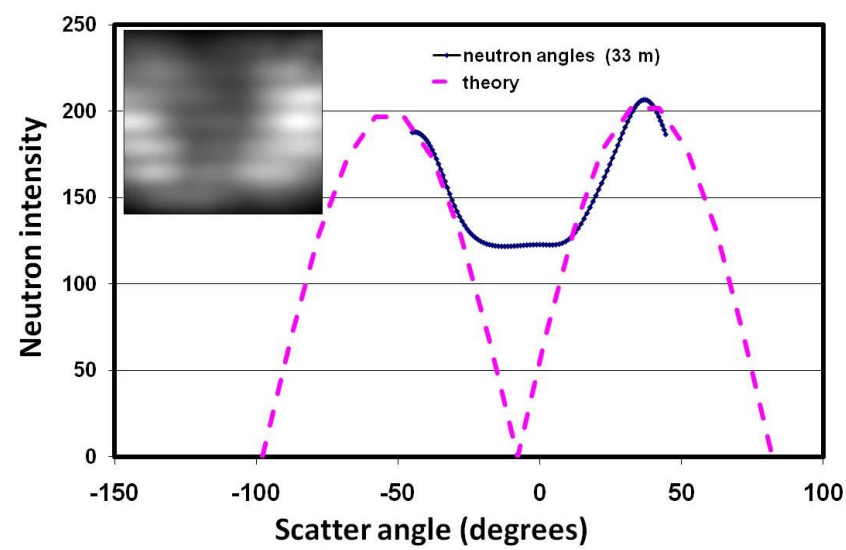

Fig. 5. Horizontal profile of neutron scattering angle distribution compared to theory. Ideally, the intensity should be zero at the center.

Using the same data, we have constructed two images of the source plane by plotting overlapping ellipses on a back- projection plane. In both cases, the ellipses are generated by the intersection of the plane with cones, the axes of which point along the measured scattered neutron directions. In one case, the angle of the cone is determined in the conventional way, from the ratio of the energy deposited in the first plane to the scattered energy determined by time of flight. In the second case, the angle of the cone is assumed to be equal to $45^{\circ}$, which is the most probable angle of scatter. The two methods agree on the location of the source but differ in contrast and profile. The latter processing method gives a sharper intensity profile and higher signal/noise.

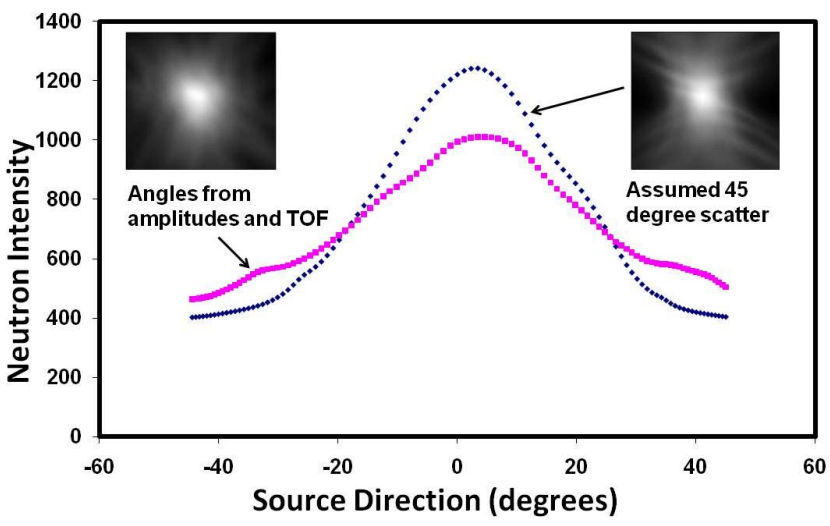

Fig. 6. Reconstructions of neutron image for source at $33 \mathrm{~m}$ using two different methods.

This comparison between the two methods becomes even more marked in data acquired at a longer range of $175 \mathrm{~m}$ where there are fewer events available to smooth out the image (see Fig. 7). It appears that combined measurements of the deposited energy and scattered energy introduce larger errors into the estimation of scattering angle than those made in simply assuming that all events have scattering angles of approximately $45^{\circ}$. This approach can be combined with selffiltering methods that emphasize the overlapping regions of the plotted ellipses [4].

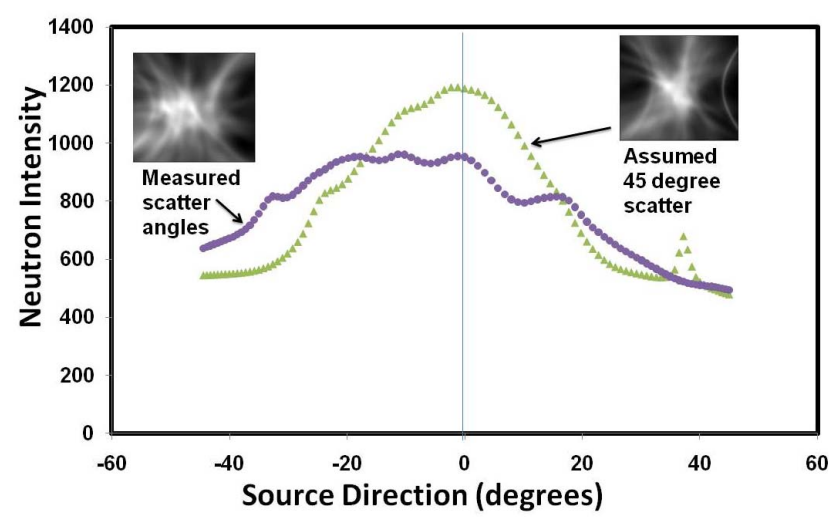

Fig. 7. Reconstructions of neutron image for the source at $175 \mathrm{~m}$ using the two methods. The two profiles were sampled horizontally across the centers of the reconstructed images.

\section{Neutron energies}

The net neutron count rate is given in Fig. 8 as a function of distance from the source. The count rate was measured in the 
neutron energy range 2-8 $\mathrm{MeV}$, with background subtracted. Monte Carlo simulations were conducted for $2 \mathrm{MeV}$ neutrons, and compared with an attenuation calculation, which is normalized to count rate and included in Fig. 8. The agreement between the data points and the calculations is very good.

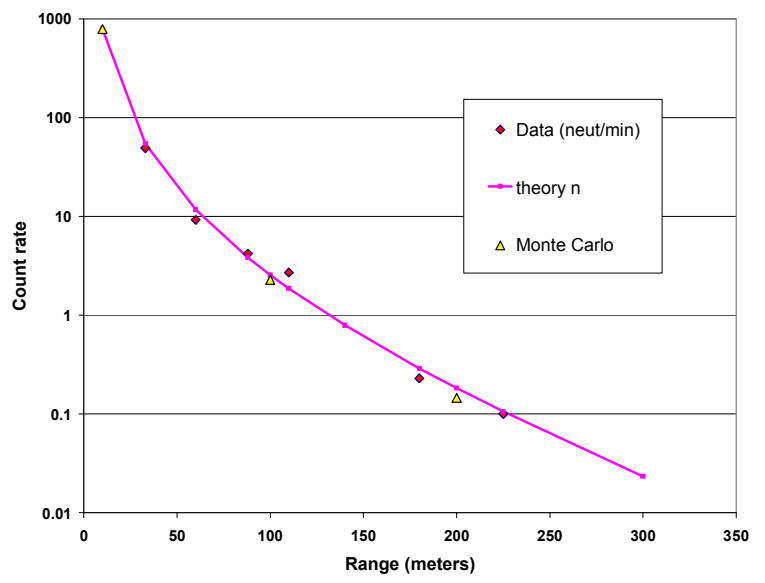

Fig. 8. Net neutron count rate as a function of distance from source.

Neutron energy spectra were determined from the pulse height in the first detection plane added to the time-of-flight calculation of energy from the interplanar coincidence time, as shown in Fig. 9. Note that the units of the ordinate axis are neutrons per second and that the ${ }^{252} \mathrm{Cf}$ spectrum peaks at 2 $\mathrm{MeV}$. The neutron energy window used for analysis includes $>80 \%$ of the total measured at 33 meters. The change in the shape of the ${ }^{252} \mathrm{Cf}$ spectra with increased range is due to the energy dependence of the interaction cross sections for neutrons with the constituents of air, which are greater at lower energies.

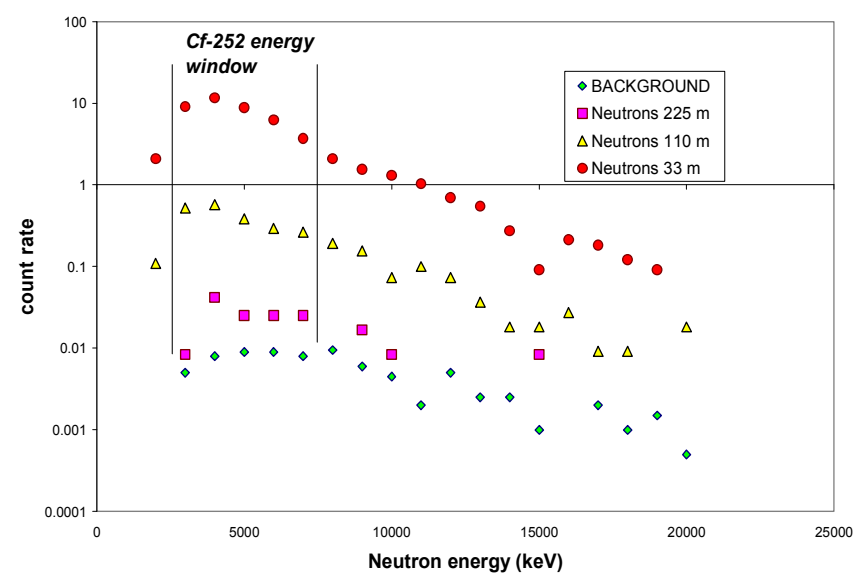

Fig. 9. Double scatter neutron spectra at various source distances.

\section{Efficiency/cosmic-ray background response}

The efficiency of the DSNS is sensitive to the threshold of the neutron response which was operated here at about 2 $\mathrm{MeV}$. Less than half the ${ }^{252} \mathrm{Cf}$ neutrons are emitted above 2
MeV. Final neutron efficiency determination awaits calibration of the neutron source.

An important factor in long range detection is the ratio of detection sensitivity to spontaneous fission neutrons (1-8 $\mathrm{MeV})$ relative to cosmic-ray neutrons $\left(10^{-8}-1000 \mathrm{MeV}\right)$, $\mathrm{R}_{\mathrm{sf} / \mathrm{cr}}$. The most common detector for fast neutrons is the moderated ${ }^{3} \mathrm{He}$ system, which is non-directional and has a wide energy response. The DSNS has an advantage in the $\mathrm{R}_{\mathrm{sf} / \mathrm{cr}}$ ratio because it is directional and rejects more than half of the isotropic neutrons from cosmic-rays, which would appear to have negative TOF. A second advantage is that the DSNS analysis window can be controlled precisely to exclude cosmic ray neutrons that are not within the energy range of spontaneous fission emission. By comparison, the moderated system may have a preferred sensitive energy range, but it is very wide. For example, all of the spectra sampling Bonner spheres [9] respond to high energy neutrons (10-1000 MeV), and to lower energy neutrons $\left(>10^{-6} \mathrm{MeV}\right)$. On the basis of the parameters of this DSNS [2], and those of the 8.2" Bonner sphere:

$$
\begin{aligned}
& \mathrm{R}_{\text {sf } / \mathrm{cr}}(\mathrm{DSNS})=8 \\
& \mathrm{R}_{\mathrm{sf} / \mathrm{cr}}(8.2 \text { ” Bonner sphere })=2.1
\end{aligned}
$$

Thus, the DSNS will have a significant advantage in signal over background when the background is from cosmic-ray neutrons. The relative response of the detectors to the neutron production in cosmic-ray irradiated dense bulk media (ship effect) is a more complicated issue. However, the ability of the DSNS to locate a bright spot in the scene is an additional factor in determining the presence of a man-made neutron emitter.

\section{CONCLUSIONS}

We have demonstrated detection of a spontaneous fission source at distances up to $225 \mathrm{~m}$ using a large-area doublescatter neutron spectrometer. Two limiting factors for longrange detection are attenuation by the air and background from cosmic ray neutrons. We tested a different analysis method that avoids accumulating errors due to amplitude measurements and found that it gave better directional information than the traditional method. An analogous method can be used to give the direction to a source of gammas, based on the most probable scattering angles. Neutron energy spectra can be used to distinguish fission from background.

\section{ACKNOWLEDGMENT}

This work was supported by U.S. Department of Energy, Office of Nonproliferation Research and Development. This manuscript has been authored by Brookhaven Science Associates, LLC under Contract No. DE-AC02-98CH1-886 with the U.S. Department of Energy. The United States Government retains, and the publisher, by accepting the article for publication, acknowledges, a world-wide license to publish 
or reproduce the published form of this manuscript, or allow others to do so, for the United States Government purposes.

\section{REFERENCES}

[1] Peter E. Vanier and Leon Forman, "An 8-element Fast-Neutron DoubleScatter Directional Detector", Proceedings of the SPIE International Symposium on Optical Science and Technology, Conference 5923, Hard X-rays, Gamma Rays and Particles, San Diego, CA, August, 2005.

[2] Peter E. Vanier, Leon Forman, Istvan Dioszegi, and Cynthia Salwen, "Design of a large-area fast neutron directional detector", Conference Record of IEEE Nuclear Science Symposium, San Diego, CA, November, 2006.

[3] Peter E. Vanier, Leon Forman, Istvan Dioszegi, Cynthia Salwen, and Vinita J. Ghosh, "Calibration and testing of a large-area fast-neutron directional detector", Conference Record of IEEE Nuclear Science Symposium, Honolulu, HI, November, 2007.

[4] Peter E. Vanier, Istvan Dioszegi, Cynthia Salwen, Vinita Ghosh and Leon Forman, "Stand-off detection of special nuclear materials using neutron imaging methods", Conference Record of IEEE Nuclear Science Symposium, Dresden, Germany, November, 2008.

[5] W.C. Sailor, R.C. Byrd, A. Gavron, R Hammock and Y. Yariv, "A neutron source imaging detector for nuclear arms treaty verification", Nucl. Sci. and Eng. vol. 109, pp. 267-277, 1991.

[6] P.D. Zecher, A. Galonsky, J.J. Kruse, S.J. Gaff, J. Ottarson, J. Wang, F. Deak, A. Horvath, A. Kiss, Z. Seres, K. Ieki, Y. Iwata, H. Schelin, "A large-area, position-sensitive neutron detector with neutron/gamma-ray discrimination capabilities", Nucl. Instrum. and Meth. A 401329 (1997)

[7] P. Marleau, J. Brennan, K. Krenz, N. Mascarenhas and S. Mrowka, "Advances in imaging fission neutrons with a neutron scatter camera", Conference Record of IEEE Nuclear Science Symposium, Honolulu, 2007.

[8] Ulisse Bravar, Paul J. Bruillard, Erwin O. Flückiger, John R. Macri, Alec L. MacKinnon, Mark L. McConnell, Michael R. Moser, James M. Ryan, and Richard S. Woolf, "Development of the Fast Neutron Imaging Telescope", Conference Record of IEEE Nuclear Science Symposium, Puerto Rico, N6-3, 107, 2005.

[9] M. S. Gordon, P. Goldhagen, K. P. Rodbell, T. H. Zabel, H. H. K. Tang, J. M. Clem, P. Bailey, "Measurement of the flux and energy spectrum of cosmic-ray induced neutrons on the ground", IEEE Trans. Nuclear Sci. v51(6), p3427, 2004.

[10] Leon Forman, P. E. Vanier, and Keith E. Welsh, "Distinguishing spontaneous fission neutrons from cosmic-ray background", Proceedings of the SPIE - Hard X-ray and Gamma-ray Detector Physics V, 5541-7, (217-225) Denver, CO., Ca., 4-5 August, 2004. 